Los saberes docentes en la mira: una aproximación polifónica. Páginas 111-130 en Revista de la Escuela de CienCIAS de LA EdUCACIÓN, AÑo 11, NÚMERo 10, ENERO A DICIEMBRE DE 2015. ISSN 1851-6297. ISSN en Línea 2362-3349.

\title{
LOS SABERES DOCENTES EN LA MIRA: UNA APROXIMACIÓN POLIFÓNICA
}

\author{
Por Andrea Alliaud* \\ Universidad de Buenos Aires, Argentina. \\ andrealliaud@ciudad.com.ar \\ Por Lea Vezub*** \\ Universidad de Buenos Aires, Argentina. \\ leitiv@gmail.com
}

Recibido: 20/06/2014 Aceptado: 27/08/2014

\section{Resumen}

Preguntarse acerca de lo que maestros y profesores tienen que saber para poder enseñar hoy, adquiere sentido en el marco de las transformaciones que atraviesan los sistemas educativos y el oficio docente. Este trabajo intenta brindar algunas respuestas a partir de los resultados obtenidos en una serie de investigaciones destinadas al estudio del "saber de la experiencia"; aquel que los docentes producen en su actividad cotidiana.

Para analizar esta problemática se ha acudido a una polifonía de voces que reúne a los especialistas, a los propios docentes y a los formadores. Si bien tanto unos como otros valoran los saberes específicos ligados a la dimensión "clásica" del oficio, llama la atención el protagonismo que cobran los saberes vinculados con los contextos y los destinatarios de la acción pedagógica. Posicionados desde el puesto de trabajo, maestros y profesores valoran principalmente aquellos saberes que les permiten "poder hacerlo". Los formadores enfatizan la importancia de relacionar la teoría con la práctica profesional. A partir de estos planteos, alertamos sobre la necesidad de orientar la formación docente hacia la transmisión del oficio (docente) y nos

* Doctora en Educación (UBA). Docente-investigadora de la Facultad de Filosofía y Letras (UBA). Profesora de Posgrado en la Universidad Torcuato Di Tella. Coordinadora del Área de Formación Docente en la OEI de Argentina. Especialista en Formación Docente.

** Doctora en Educación (UBA). Docente e Investigadora de la Facultad de Filosofía y Letras (UBA). Profesora Adjunta Regular de Metodología de la Investigación Educativa, Dpto. de Humanidades, Universidad Nacional de Moreno. Consultora internacional, especialista en formación y desarrollo profesional Docente. 
Revista de la Escuela de Ciencias de la Educación, año 11, número 10, enero a diciembre de 2015. Páginas 111-130. ISSN 1851-6297. ISSN EN LINEAA 2362-3349. LOS SABERES DOCENTES EN LA MIRA: UNA APROXIMACIÓN POLIFÓNICA. ANDREA Alliaud - Lea Vezub

interrogamos por el "más allá" de la contextualidad, la situacionalidad y la subjetividad tan mentadas en estos tiempos.

\title{
Palabras clave:
}

Formación docente - Saberes docentes - Experiencia - Oficio.

\begin{abstract}
Wondering about what teachers should know to teach nowadays makes sense within the framework of transformations that undergo educational systems and teaching. This research intends to offer answers from the results obtained in a series of studies focused on "knowing from the experience", knowledge demonstrated by teachers in their everyday experience.

In order to analyze this problem, polyphony of voices that represent specialists, teachers and trainers has been considered. Even though all of them value the specific knowledge associated to the classic dimension of the craft, it is striking the importance given to the knowledge related to contexts and target audience of the pedagogical action. From their work position, teachers greatly value those sets of knowledge that allow "the ability of doing it". Trainers highlight the importance of linking theory with professional practice. Considering this, we alert to be attentive to the need of orientating teaching training to the transmission of (teaching) trade and we query "beyond" contextuality, situationality and subjectivity deeply discussed these days
\end{abstract}

\section{Key Words:}

Teaching training - Teaching knowledge - Experience - Craft.

\section{Introducción}

La formación docente nunca ha sido tarea sencilla. Pero en la actualidad se han complejizado y renovado las demandas y desafíos que enfrenta, debido a las mutaciones que atraviesa el oficio. Los avances tecnológicos y la rápida transformación de los escenarios actuales interpelan a los sistemas educativos tradicionales y a sus agentes; por lo tanto, se hace necesario revisar cuáles son los saberes y procedimientos necesarios para formar docentes que sepan y puedan enseñar hoy.

La problemática de los saberes docentes ocupa el centro de la escena y su debate se renueva al tratar de mejorar la formación de los profesores. En primer lugar, porque el conocimiento y el saber son elementos clave de la acción educativa y de la tarea de los docentes, constituyen parte de la materia prima con la que estos trabajan. En segundo lugar, para relacionarse con los estudiantes y lograr que estos orienten su aprendizaje hacia el currículum escolar, los docentes tienen que dominar ciertas estrategias y medios propios, fundados en el conocimiento de la pedagogía, de la didáctica, del currículum y de la comunicación. En tercer lugar, los acelerados cambios sociales, las 
Revista de la Escuela de Ciencias de la Educación, año 11, número 10, enero a diciembre de 2015. PÁginas 111-130. ISSN 1851-6297. ISSN EN LINEA 2362-3349. LOS SABERES DOCENTES EN LA MIRA: UNA APROXIMACIÓN POLIFÓNICA. ANDREA AlLiaud - Lea VezuB

transformaciones en las formas de producción han colocado al conocimiento -y sobre todo a la capacidad de acceder, buscar y generar nuevos saberes-en un lugar de privilegio y de poder para el ejercicio de la ciudadanía y los derechos.

El vehículo principal de la acción docente, el objeto de la enseñanza es la construcción y circulación de saber, de conocimiento. Por ello recuperar el valor de la escuela, implica poner en primer plano a la enseñanza y a la transmisión, entendida de manera no unidireccional, ni mecánica. Como sostiene Hassoun (1996) la aventura propia de la transmisión es que somos diferentes de nuestros predecesores y también de nuestros descendientes, por lo que una transmisión "bien lograda" es aquella que ofrece libertad, es decir, permite que los otros hagan con lo que les transmitimos un camino sensiblemente diferente al nuestro. Se afirma que la escuela está en crisis y sostenemos que parte de esa crisis tiene que ver con los procesos de transmisión (1) y de enseñanza, de allí la necesidad de revisar los saberes que circulan en el sistema escolar y en las instituciones de formación docente. Si dicha tarea ha mutado notablemente, como todos afirman, es probable que las bases del saber formalizado y transmitido en los espacios de formación docente hoy resulten insuficientes, así como el aprendizaje práctico y la socialización profesional entendida como mera acumulación de años. De allí que resulta pertinente indagar de qué manera los docentes hoy día "se las arreglan" para seguir enseñando, ¿qué saberes valoran, a qué conocimientos recurren, cómo los jerarquizan?

Las soluciones estándar se muestran insuficientes; las prácticas de enseñanza no se resuelven exclusivamente a través de la pericia técnica y del conocimiento estandarizado. Hoy más que nunca se requiere que los profesores puedan crear, probar, experimentar e innovar, "construir su experiencia" mientras enseñan (Alliaud, 2012). Lo que los profesores saben a nivel declarativo, teórico, no se transforma, ni se refleja automáticamente en su acción, en sus prácticas de enseñanza (Calderhead, 1988). En este sentido Sennett (2009) señala que el saber hacer del "virtuoso" es mucho más que aplicar una técnica o un conocimiento especializado; es poder solucionar problemas, descubrir nuevos territorios, revisar los supuestos que sostienen nuestras acciones y decisiones. Esto implica comprometerse con lo que se hace, y, fundamentalmente, ser capaz de conjugar el pensamiento con la acción.

Las propuestas y las prácticas formativas intentan expresar los conocimientos, destrezas y habilidades que se supone los maestros y profesores necesitan "adquirir" para poder enseñar. Es frecuente que a la hora de formular un plan de formación se seleccionen contenidos, se jerarquicen, se enuncien capacidades, destrezas o competencias y habilidades en "abstracto", sin conocer profundamente de qué se trata o en qué consiste la práctica profesional (2). La formación (inicial y continua) pretende "regular" un saber "de oficio" escurridizo, difícil de generalizar, ya que al hacerlo se eliminan la particularidades puestas en juego en todo acto de enseñanza. Por otro lado, los docentes -al igual que 
Revista de la Escuela de Ciencias de la Educación, año 11, número 10, enero a diciembre de 2015. Páginas 111-130. ISSN 1851-6297. ISSN EN LINEAA 2362-3349. LOS SABERES DOCENTES EN LA MIRA: UNA APROXIMACIÓN POLIFÓNICA. ANDREA AlLiaud - Lea Vezub

otros profesionales- producen en su quehacer un saber que, a pesar de su potencialidad para dar respuesta a las situaciones prácticas, no se incorpora en los ámbitos de formación.

Indagar la naturaleza de la práctica de enseñar y los saberes que en ella se ponen en juego, es una inquietud que como investigadores y formadores nos preocupa al entender que esta vía puede conducir a definir y desarrollar propuestas formativas que dejen a los docentes mejor provistos para enfrentar los desafíos y las complejidades que asume la enseñanza en el presente. Desde esta perspectiva, venimos desarrollando una serie de proyectos de investigación (3) dirigidos a profundizar en las dimensiones del saber profesional de los docentes considerando diferentes perspectivas, fuentes, voces y aproximaciones metodológicas.

La investigación que en esta oportunidad presentamos, se propuso focalizar particularmente en la manera en que los profesores perciben y definen su actividad en sus contextos. De manera ambiciosa este artículo intenta reunir en una misma discusión, los avances alcanzados en diversas instancias del trabajo de campo, con la intención de poner a dialogar los hallazgos obtenidos a partir de: (i) la revisión de la literatura especializada; (ii) la perspectiva de docentes (de nivel primario y secundario); y (iii) la mirada de los formadores, particularmente de quienes están ligados a los espacios curriculares del campo de la formación práctica profesional.

En una primera etapa del Proyecto, se utilizó un instrumento que recogió de manera estandarizada, información sobre los docentes, sus opiniones referidas a la función de la escuela, al quehacer docente, a los saberes indispensables para su desempeño y los sentimientos de los profesores frente a su tarea (Alliaud y Vezub, 2012) (4). En un segundo momento, se abordó en mayor profundidad los saberes que los docentes consideran "valiosos" para enseñar. En lugar de indagar directamente sobre ellos, se optó por acceder a relatos de situaciones de enseñanza en clases bien y mal avenidas. Aquello que "salió bien" y lo que no, fue recuperado y narrado por los docentes, tanto a nivel descriptivo como interpretativo de lo que ocurrió. ¿Qué hacen o hicieron aquellos a los que la enseñanza les resultó? ¿Cómo explican o dan cuenta de aquello que efectivamente aconteció? En síntesis, interesó reparar en el quehacer de maestros y profesores para, desde allí, develar los saberes que estos "haceres" implican. Por último, la tercera etapa del trabajo de campo incorporó la perspectiva de los formadores y de los expertos -a través de la literatura especializada-con la finalidad de analizar y poner en tensión los saberes valorados por los docentes y aquellos que se plasman en modelos teóricos, así como los que fundamentan las prácticas de formación (5). 
Revista de la Escuela de Ciencias de la Educación, año 11, número 10, enero a diciembre de 2015. PÁginas 111-130. ISSN 1851-6297. ISSN EN LINEA 2362-3349. LOS SABERES DOCENTES EN LA MIRA: UNA APROXIMACIÓN POLIFÓNICA. ANDREA Alliaud - Lea Vezub

\section{La voz de los especialistas}

Existen numerosas categorizaciones que por diversos motivos no terminan de responder satisfactoriamente a la pregunta acerca de qué debe saber un docente y por qué. Las clasificaciones que retoma el trabajo de Terigi (2012), al igual que la propia revisión realizada sobre la bibliografía de décadas anteriores correspondiente a los años ochenta y noventa (Bromme, 1988; Gimeno Sacristán, 1987; Schulman, 1987; Elbasz, 1983), muestran que existen algunas coincidencias en torno a los siguientes grupos de saberes y conocimientos que formarían parte del "background" de los enseñantes:

1. el contenido a enseñar, los saberes disciplinares

2. Ios aprendices, sus características referidas al desarrollo psicológico, cultural, social, etc.

3. el currículum y los propósitos educativos, fines y valores educativos

4. el conocimiento pedagógico, didáctico para enseñar, evaluar a los alumnos y gestionar el aula.

En esta última categoría ciertos autores distinguen entre conocimiento pedagógico / educativo general y conocimiento pedagógico de los contenidos disciplinares, es decir, las denominadas didácticas específicas (Ávalos, 2009; Terigi, 2009). Mientras que otros especialistas circunscriben el conocimiento pedagógico a lo curricular y a los fines educativos. El trabajo de Bromme (1988), difundido en los inicios de este debate, ya formulaba el problema en términos bastante similares a los planteamientos actuales:

Los conocimientos profesionales de los profesores han sido hasta ahora asombrosamente poco estudiados. De cualquier modo entre los profesores está extendida la opinión de que sus conocimientos profesionales no constan únicamente de lo que una vez aprendieron en su formación teórica. Así, pues, se dice a menudo a los enseñantes que comienzan su carrera profesional que su saber teórico no es especialmente útil para la práctica cotidiana. ¿Qué papel juega entonces el saber teórico y de qué tipo son los conocimientos profesionales? (...) es deseable disponer de conocimientos acerca de en qué consiste el saber profesional de los enseñantes y cómo este se relaciona con los conocimientos de la asignatura, la pedagogía y de la psicología (Ibid: 19).

La revisión de la literatura más actual muestra que a los grupos o conjuntos de conocimientos ya mencionados y reconocidos por los expertos desde hace varias décadas, recientemente se han agregado los siguientes:

5. las tecnologías de la información y la comunicación

6. las estrategias para educar en la diversidad y ligado a lo anterior, 
Revista de la Escuela de Ciencias de la Educación, año 11, número 10, enero a diciembre de 2015. Páginas 111-130. ISSN 1851-6297. ISSN EN LINEAA 2362-3349. LOS SABERES DOCENTES EN LA MIRA: UNA APROXIMACIÓN POLIFÓNICA. ANDREA Alliaud - Lea Vezub

7. el conocimiento de los contextos en que se desenvuelven las prácticas educativas (incluyendo los niveles institucional y comunitario de los alumnos, además del sistema educativo y social general).

A pesar de haberse acumulado mayor evidencia e investigación sobre la cuestión, en particular sobre los denominados conocimientos o esquemas prácticos proveniente de los estudios sobre el pensamiento del profesor y los razonamientos prácticos (Pendelbury, 1998), la pregunta continúa sin resolverse. Probablemente no avanzaremos si pretendemos dar una respuesta certera y duradera que elimine las ambigüedades y expectativas siempre renovadas y cambiantes que las sociedades y los sistemas educativos depositan en la formación, al tratar de contar con docentes que puedan enseñar a todos los alumnos, enfrentar cualquier situación, en cualquier contexto. La teoría y la investigación han demostrado que la pedagogía dista de ser una ciencia tan racional y metódica como algunos han pretendido, ya que en la práctica de la enseñanza lo que prima es la evaluación de la situación singular, lo mutable, las contradicciones e incertidumbres que demandan un equilibrio entre los principios y la lectura atenta, donde la percepción de los detalles desempeña un papel clave aunque difícil de ser entrenado y esquematizado.

Del trabajo de Bromme nos interesa retomar en primer lugar, la idea -ampliamente aceptada por la comunidad de expertos- acerca de que existe algo más que conocimiento teórico y formal en el saber y desempeño profesional docente. Un conocimiento vinculado al "saber hacer", a la experiencia que se adquiere en el devenir de la práctica. En segundo lugar, se encuentra el planteo de la especificidad de un saber docente que lejos de agotarse en el conocimiento pedagógico y en las disciplinas del currículum, posee una singularidad y rasgos propios a partir de los cuales se constituye y se relaciona con otros campos o dominios del saber, tales como la pedagogía, las ciencias de la educación, la psicología educacional, etc.

En este terreno el trabajo de Maurice Tardif (1991) brinda algunas explicaciones que pueden orientar en la búsqueda de nuevas respuestas. Para el autor, los educadores definen su práctica -la transmisión de saberes socialesde una o de otra manera, por la relación que ellos tienen con los saberes que poseen y trasmiten. No obstante, la naturaleza de esa relación es problemática, al igual que la definición de los saberes puestos en juego. ¿Los docentes solo trasmiten saberes producidos por otros? ¿En el transcurso de su tarea producen otros saberes que les son propios? ¿Dónde y cómo se aprenden estos saberes? ¿Es posible capturarlos, sistematizarlos, formalizarlos para utilizarlos en procesos y dispositivos de formación inicial y permanente de los docentes? ¿Los especialistas, formadores y docentes en actividad coinciden a la hora de jerarquizar y valorar los saberes que son útiles y necesarios para la enseñanza?

Una de las respuestas dadas por Tardif y otros autores (Terigi, 2012; Alliaud, 2012) es que en su práctica los profesores producen un saber que, por lo gene- 
Revista de la Escuela de Ciencias de la Educación, año 11, número 10, enero a diciembre de 2015. PÁginas 111-130. ISSN 1851-6297. ISSN EN LINEA 2362-3349. LOS SABERES DOCENTES EN LA MIRA: UNA APROXIMACIÓN POLIFÓNICA. ANDREA AlLiaud - Lea VezuB

ral, no es reconocido, legitimado ni valorado como el saber experto o formalizado (ni aún por los mismos docentes). Al enseñar, los docentes integran diverso tipo de saberes, considerados en un sentido amplio (conocimientos, competencias, habilidades y actitudes); es decir el saber, el saber hacer y el saber ser (Tardif, 2000). Más concretamente, se trata de saberes: profesionales, de las ciencias de la educación y pedagógicos; disciplinares; curriculares y; de la experiencia, fundados en su trabajo cotidiano con los alumnos y en el conocimiento del medio. Dubet (2006) caracteriza a los profesores como bricoleurs que, con el paso de los años van construyendo sus métodos, sus modos de actuar y sus hábitos. El autor señala que los profesores no suelen dar cuenta de estos "secretos de fabricación" ni en las entrevistas ni en los espacios de intercambio formales. Estas formas, semejantes a las recetas de cocina, se elaboran en el transcurrir de la propia experiencia y se caracterizan por mezclar distintos ingredientes que se prueban y validan o no en función de lo que sucede (Alliaud, op. cit.). De este modo, se constituye una amalgama plural de saberes que puede resultar más o menos coherente y a partir de la cual se asume el trabajo cotidiano, las tareas de enseñanza y se consolida la identidad profesional.

Además de la pluralidad, interesa destacar dos elementos de estos saberes: el primero es la heterogeneidad de los ámbitos en los cuales se adquieren, trasmiten, circulan y producen. Nos referimos a las instituciones de formación inicial, a las escuelas de práctica, a las escuelas donde los noveles se insertan, a la práctica individual y colectiva, a la formación continua, a la búsqueda ocasional, aislada, que el docente realiza frente a un problema concreto y singular o a la búsqueda sistemática que emprende a lo largo de su trayectoria (Vezub, 2011) como parte de su desarrollo profesional, compromiso y reflexión permanente. El segundo, es la relación que los docentes establecen con estos tipos de saber. Con los saberes profesionales, curriculares y disciplinares mantienen una relación de "exterioridad", basada en la división del trabajo y las relaciones de poder existente entre expertos, científicos, intelectuales, e investigadores; entre universidades / institutos y escuelas. Mientras que con los saberes de la experiencia mantienen una relación que puede caracterizarse en términos de "interioridad" (Tardif, 1991). La posibilidad de mejorar su práctica, la orientación que esta adquiera, dependen en gran medida de la capacidad que los docentes tengan para integrar, sistematizar y movilizar estos saberes de la experiencia, de allí que su identificación y sistematización resulte clave para los procesos de formación y desarrollo profesional.

La bibliografía, especialmente la producción derivada de la investigación acerca del pensamiento del profesor y del paradigma ecológico del aula, centrada en la indagación de las interacciones y en los procesos internos del enseñar y el aprender, ha establecido la existencia de otro elemento. Frente a la evidencia de que lo que el profesor sabe acerca de la enseñanza no se traduce automáticamente en "acción" y en prácticas educativas coherentes 
Revista de la Escuela de Ciencias de la Educación, año 11, número 10, enero a diciembre de 2015. Páginas 111-130. ISSN 1851-6297. ISSN EN LINEAA 2362-3349. LOS SABERES DOCENTES EN LA MIRA: UNA APROXIMACIÓN POLIFÓNICA. ANDREA Alliaud - Lea Vezub

con dicho saber, que lo que sucede en las clases es en gran parte imprevisto, impredecible y situacional. A partir de estas evidencias comenzó a hablarse de los procesos metacognitivos y la reflexión de y sobre la práctica (Schön, 1992) como un saber propio de los docentes y una habilidad que no siempre se encuentra y, en consecuencia, es preciso desarrollarla sistemáticamente durante la formación inicial y continua, para incorporarla al perfil docente. Todas las fuentes consultadas y la literatura que aportan los expertos sobre la formación docente coinciden en la idea del docente reflexivo, investigador de su práctica, derivada de los clásicos trabajos de Stenhouse, Schön y Elliot. No obstante, todavía no es tan clara la evidencia acerca de cómo lograrlo, cuáles son los dispositivos formativos necesarios, es decir qué saberes y habilidades en este caso deberían poseer los formadores para habilitar estas capacidades en los futuros docentes. Otras veces, las condiciones institucionales, de la organización y trabajo de los profesores obstaculizan la implementación de este tipo de prácticas y dispositivos ya que requieren gran inversión de tiempo y recursos humanos para acompañar a los maestros y profesores en formación.

Este componente propio del saber profesional docente permite no solo comprender, interpretar las dinámicas del aula y adaptar las estrategias a las necesidades y características de los grupos de estudiantes concretos, sino que además regula la actividad cognitiva del docente, al promover la mejora y cambio permanente de su práctica, sentando las bases de su desarrollo profesional. Se trata en definitiva de aprender de la propia experiencia. La práctica educativa puede $-\mathrm{y}$ de hecho en muchas ocasiones así sucede- funcionar al margen del conocimiento formal, teórico y científico derivado de las ciencias de la educación. Por otra parte la práctica, el desempeño docente, rebasa los límites del conocimiento formal, constituido y validado, no es deseable ni posible circunscribir la acción docente a lo que dicen los expertos, la investigación y la teoría. Por ello, la adquisición de conciencia acerca de la práctica, de los propios esquemas prácticos adquiridos y la posibilidad de aprender de la experiencia propia y ajena, debería convertirse en una idea fuerza tanto en la formación inicial como permanente.

Para Gimeno Sacristán (1997) los docentes disponen de una serie de esquemas prácticos para ordenar y orientarse en la acción, son rutinas para la práctica (más o menos flexibles según los casos) que facilitan el día a día de la clase, proporcionan cierto orden y progreso, constituyen parte del saber hacer, del oficio y arte del docente. En su mayoría estos esquemas han sido aprendidos no tanto en la formación inicial, sino, fundamentalmente en el propio contexto escolar. Es por ello que pueden actuar muchas veces reproduciendo el statu quo, la gramática escolar y las formas instituidas de enseñar y de aprender.

En clave más socioeducativa es interesante revisar la clasificación propuesta por Cecilia Braslavsky (1999) al pensar el lugar de los maestros y profesores en América Latina y el proceso de desprofesionalización técnico e 
Revista de la Escuela de Ciencias de la Educación, año 11, número 10, enero a diciembre de 2015. PÁginas 111-130. ISSN 1851-6297. ISSN EN LINEA 2362-3349. LOS SABERES DOCENTES EN LA MIRA: UNA APROXIMACIÓN POLIFÓNICA. ANDREA AlLiaud - Lea VezuB

intelectual que atravesaron en décadas anteriores. La autora señala dos errores comunes entre quienes pretenden profesionalizar a los docentes y dotarlos de un nuevo perfil más acorde a los contextos y nuevas necesidades de los sistemas educativos. El primero es la "sobresimplificación" del problema, al decir que los docentes deben saber y saber enseñar. El segundo es la "falta de jerarquización" que se observa en la presentación de extensos listados de cualidades y conocimientos que los profesores deberían poseer y no poseen. La alternativa para Braslavsky es pensar en una identidad docente "múltiple y consistente", una propuesta clara, simple y comunicable; capaz de reemplazar a la propuesta fundacional del magisterio, basada en cinco dimensiones fundamentales: pedagógica didáctica; institucional; productiva; interactiva y la dimensión de la especificación.

\section{La voz de los docentes}

Al hablar de su enseñanza, los docentes que participaron del estudio consideran que las buenas o malas clases suceden debido a la motivación, participación, interés o "enganche" que demuestran los alumnos:

"... la experiencia que más recuerdo la llevé adelante con los alumnos de tercer año de otra escuela, con ellos estábamos viendo Invasiones inglesas y la verdad notaba que los chicos no se estaban enganchando, veía como que se dormían, si bien no generaban problema en clase, es como que no estaban enganchados. Entonces se me ocurrió hacer una puesta en escena..." (P6, Profesora Secundaria de Historia).

Contenidos que les interesen, formas de trabajo, recursos que los motiven y logren captar su atención, "clases atractivas", son los rasgos que aparecen recurrentemente cuando los docentes relatan sus experiencias de enseñan$z a$. Muchas veces estas clases abordan temas de actualidad, vinculados con el presente o el pasado reciente (derechos humanos, genética, la dictadura militar, la vida de los jóvenes de una villa, los valores, etc.). También utilizan nuevas tecnologías, imágenes o dispositivos digitales. Asimismo, aunque en menor medida, consideran que el éxito o el fracaso en sus clases se deben a la planificación, a la selección de contenidos que se realizó y a su grado de adecuación al grupo, aspectos se visualizan en la organización, clima y buen transcurrir de la clase. Parte del éxito del trabajo docente parece descansar en estos saberes "pedagógicos-didácticos" y el parámetro para medirlo es: su adecuación a los intereses de los alumnos. La capacidad para afrontar los problemas de aprendizaje, el reconocimiento de sus ideas previas y conocer el mundo en el que viven, complementan los anteriores. Los docentes comentan que las clases "salen bien" cuando: 
Revista de la Escuela de Ciencias de la Educación, año 11, número 10, enero a diciembre de 2015. Páginas 111-130. ISSN 1851-6297. ISSN EN LINEAA 2362-3349. LOS SABERES DOCENTES EN LA MIRA: UNA APROXIMACIÓN POLIFÓNICA. ANDREA Alliaud - Lea Vezub

"Se dieron las condiciones, primero porque había material, se indagó antes sobre lo que ellos sabían. Y a partir de eso se armó la clase, el juego" (P12, Maestra de Primaria, $1^{\circ}$ Ciclo).

"Generalmente la clase planificada es como que surge bien, llegas al final de la clase y estas conforme con lo que diste, sabes que lo que hiciste estaba bien. No es lo mismo venir sin nada" (P5, Maestra de Primaria, $2^{\circ}$ ciclo).

"Bueno, para la escuela real de hoy en día es necesario plantarse, mucha actitud. No es lo mismo enseñarle a los chiquitos que a quinto, sexto. Es necesario ver el mundo de los chicos, no olvidarse que alguna vez nosotros también fuimos chicos. Entonces me parece que eso es crucial (P7, Maestra de Primaria, $1^{\circ}$ ciclo).

Cuando se les pregunta a los docentes por aquello que "no ha salido" bien, nuevamente las respuestas aluden a las dificultades de la planificación, la selección de los contenidos o de las estrategias; en tanto demostraron ser inadecuadas para el grupo, o bien cuando el grupo no responde a lo que el docente esperaba según lo planificado. Antes que en su propia pericia profesional, el foco vuelve a estar colocado en los alumnos. En este sentido, "la clase falla" cuando:

"El docente cree que los alumnos tienen unos conocimientos previos que en realidad no tienen (...) Se realiza una planificación cerrada sin diagnosticar los saberes previos con los que los alumnos cuentan" (P5, Maestro y Profesor de Informática).

"Las estrategias que se planificaron son inadecuadas" (P13, Profesora de Filosofía, Secundaria).

"Si las estrategias no los incentivan, el grupo no responde" (P14, Maestra Primaria, $1^{\circ}$ ciclo).

En un primer nivel de análisis se puede notar en estas apreciaciones, que según los docentes, estos tendrían que:

- Saber motivar, interesar, entusiasmar, "provocar" estados en los alumnos.

- Saber planificar, seleccionar los contenidos, las estrategias y adecuarlos a ellos.

En otros relatos (pocos) se puede apreciar que los docentes -en lugar de hablar de sus alumnos- aluden a saberes, características y cualidades propias de su condición docente. Desde esta perspectiva, resultaría importante que sepan y sean de determinada manera a fin de lograr llevar adelante su tarea. En estos relatos se menciona a la formación o capacitación con la que cuentan: "Creo que lo más importante es que nunca debés quedarte quieto, siempre hay cosas que aprender, no hay que relajarse jamás. Porque si te re- 
Revista de la Escuela de Ciencias de la Educación, año 11, número 10, enero a diciembre de 2015. PÁginas 111-130. ISSN 1851-6297. ISSN EN LINEA 2362-3349. LOS SABERES DOCENTES EN LA MIRA: UNA APROXIMACIÓN POLIFÓNICA. ANDREA AlLiaud - Lea VezuB

lajas, si te confías perdiste, te volvés un profesor del montón, que va a esperar que pasen las horas y que llegue fin de mes" (P6, Profesor de Historia). También se considera que es importante el conocimiento de la materia que enseñan o el interés que el profesor demuestra sobre el tema abordado: "sobre todo un docente tiene que conocer bien lo que va a enseñar (P6, Profesora de Historia). Saber trabajar en equipo y adaptarse a los cambios "que vienen desde arriba en cuanto al enfoque educativo actual" (P4, Maestra de $\left.1^{\circ} \mathrm{Ciclo}\right)$, constituyen para otros entrevistados, nuevos desafíos del oficio que los docentes reconocen y valoran por su propia experiencia: "por trabajar con compañeras que me ayudaron a ver otras cosas. Por escuchar, por compartir, uno aprende del otro" (P4, Maestra Primaria, $1^{\circ}$ ciclo). Por último un profesor plantea que el estado de ánimo es un factor que incide en cómo sale el trabajo, hay que "estar en equilibrio con uno mismo":

"El año pasado realicé el curso del "arte de vivir" y ahí aprendí a estar en equilibrio conmigo mismo. Aprendí técnicas de respiración que me ayudaron a conservar mi voz, aprendí a relajarme para no tener que recurrir constantemente al grito" (P6, Profesor Secundario de Historia).

El análisis muestra que cuando los docentes hablan de sus clases enfatizan el saber motivar, provocar, "suscitar" ciertos estados en los alumnos. Es la enseñanza adecuada, adaptada, "a medida" del destinatario. Tal apreciación es consistente con las respuestas que maestros y profesores brindaron en el cuestionario estructurado ante la pregunta acerca de "lo que no pueden dejar de hacer para enseñar mejor". Las opciones más elegidas fueron: "conocer a los alumnos" y "tener en cuenta el contexto de la comunidad, de la escuela". El $47 \%$ de los docentes escogieron estas respuestas. En tercer lugar, el 18\% sostiene que para realizar una buena enseñanza hay que "mantener el interés y la curiosidad intelectual" de los alumnos. Las opciones vinculadas con el saber formal (disciplinar, didáctico y pedagógico) y con el saber de la experiencia, no fueron tan valoradas por los docentes.

La investigación realizada por Antelo (2009) con profesores secundarios de la ciudad de Buenos Aires arrojó resultados coincidentes, al mostrar que los saberes que los docentes valoran en su trabajo remiten al vínculo con los alumnos. Estos saberes o habilidades para poder relacionarse con los jóvenes exigen, según los profesores, conocimiento de los sujetos (adolescentes) del presente, del mundo contemporáneo y de las nuevas tecnologías. Otros saberes que los docentes mencionan son: dar lugar a la polémica, a la opinión del otro, saber manejar a los padres y estar abierto a los cambios. Para estos profesores, además del conocimiento formalizado (didáctico, de las disciplinas) es la experiencia, la fuente privilegiada donde se adquieren las destrezas y habilidades para "pararse" frente al curso, la experiencia es la base para aprender el trato con y hacia el otro. 
Revista de la Escuela de Ciencias de la Educación, año 11, número 10, enero a diciembre de 2015. Páginas 111-130. ISSN 1851-6297. ISSN EN LINEA 2362-3349. LOS SABERES DOCENTES EN LA MIRA: UNA APROXIMACIÓN POLIFÓNICA. ANDREA Alliaud - Lea Vezub

\begin{tabular}{|l|c|c|c|}
\hline $\begin{array}{l}\text { Un profesor para enseñar mejor } \\
\text { no puede dejar de... }\end{array}$ & $\begin{array}{c}\text { Docentes } \\
\text { Primarios }\end{array}$ & $\begin{array}{c}\text { Docentes } \\
\text { Secundarios }\end{array}$ & TOTAL \\
\hline Conocer a sus alumnos & 27,5 & 22,4 & 24,7 \\
\hline $\begin{array}{l}\text { Tener en cuenta el contexto, la } \\
\text { comunidad de la escuela }\end{array}$ & 20,8 & 23,1 & 22,1 \\
\hline $\begin{array}{l}\text { Mantener el interés y la curiosidad } \\
\text { intelectual }\end{array}$ & 20,8 & 15,4 & 17,9 \\
\hline Aprender de la experiencia & 10,4 & 10,5 & 10,5 \\
\hline Estudiar la disciplina que enseña & 6,7 & 11,5 & 9,3 \\
\hline $\begin{array}{l}\text { Estudiar la didáctica de la } \\
\text { disciplina que enseña }\end{array}$ & 7,9 & 8,7 & 8,4 \\
\hline $\begin{array}{l}\text { Asumir una posición sobre los } \\
\text { problemas contemporáneos }\end{array}$ & 3,3 & 5,2 & 4,4 \\
\hline Estudiar pedagogía & 2,1 & 2,8 & 2,5 \\
\hline Otra & 0,4 & 0,3 & 0,4 \\
\hline Total (*) & 100,0 & 100,0 & 100,0 \\
\hline
\end{tabular}

Fuente: Trabajo de Campo UBACyT, Año 2011 / Alliaud y Vezub (2012)

\section{La voz de los formadores}

Una de las cuestiones que primero llama la atención es que los formadores de los Institutos plantean de manera directa y enfática que los futuros docentes deben conocer las Nuevas Tecnologías y los diversos contextos para poder trabajar con alumnos distintos. Estos aspectos se encuentran además en sintonía con las propuestas y nuevos saberes que son mencionados por los expertos y los docentes. El dominio de las nuevas tecnologías también aparece en íntima relación con el conocimiento de los sujetos, ya que constituye una de las características de los alumnos formar parte de los denominados "nativos digitales", tener esa "empatía tecnológica", frente a la que los adultos y profesores quedan descolocados. Asimismo, para los formadores "saber de tecnologías" es un emergente de las nuevas políticas educativas, de los cambios introducidos en los planes de la formación docente:

"Una cuestión que está apareciendo mucho y que antes no aparecía y que por suerte está incorporada a la currícula del profesorado, es el manejo de las nuevas tecnologías (...) aparece como fuerte por- 
Revista de la Escuela de Ciencias de la Educación, año 11, número 10, enero a diciembre de 2015. PÁginas 111-130. ISSN 1851-6297. ISSN EN LINEA 2362-3349. LOS SABERES DOCENTES EN LA MIRA: UNA APROXIMACIÓN POLIFÓNICA. ANDREA AlLiaud - Lea VezuB

que los chicos tienen ahora en las escuelas las netbooks y la verdad es que en algunos establecimientos no se puede aprovechar porque los docentes no cuentan con los conocimientos como para poder utilizar esa herramienta... es imprescindible un conocimiento de las nuevas tecnologías." (F2)

"Los alumnos y los niños incorporan nuevos saberes que hoy llamamos TIC dentro del aula, donde esto que es familiar para los chicos en los espacios lúdicos se transforma en un propósito de enseñanza. Bueno, esto es parte de los saberes dinámicos en nuevos contextos políticos, socioeconómicos, en un mundo globalizado." (F7).

En cualquiera de los dos casos lo que la formación intenta evitar es que los docentes queden al margen de lo que se supone constituye un saber familiar, común, cotidiano para los alumnos. La necesidad de estar al día se impone y la tecnología no es la excepción, sino al contrario, uno de los pasos fundamentales para lograrlo.

Por su parte, el conocimiento del contexto plantea varios retos que señalan los formadores. Por un lado, se trata de formar un docente flexible, abierto a los cambios, capaz de adaptarse a situaciones nuevas e imprevistas, de abordar emergentes; que pueda enseñar a estudiantes cultural y socialmente diversos, en instituciones variadas, cada vez más complejas y difíciles:

"Enfrentar la multiplicidad de cuestiones que encuentran en la escuela y que hacen a lo contextual, ¿no? Mucha violencia, cuestiones de intervenciones que tienen que hacer con padres con niños en contextos muy adversos, mucho asistencialismo metido en las escuelas y a veces pocas herramientas para trabajar con las dificultades que aparecen y que son cada vez más grandes" (F2).

"Esa es la dinámica que para nosotros necesita saber un docente. Que es dinámico, que es provisorio, y eso implica un empuje a los docentes noveles a que su formación de grado termina para comenzar y no cesa nunca. Es el desafío de decir 'siempre tengo cosas que aprender', porque los paradigmas van cambiando y los contextos son diversos (...) Quiero un egresado que atienda a la diversidad y que a su vez construya un aula democratizadora, que democratice el saber y logre la inclusión de todos" (F7).

De este modo, para los formadores no se trata sólo de identificar ciertos saberes válidos y necesarios, sino de reparar en las cualidades y el tipo de vínculo que se establece con esos saberes. Incluso es probable que dichos rasgos o vínculos, sean hoy en día más importantes que el saber mismo. Es por ello que mencionan qué debe conocer un docente pero también se refieren al tratamiento del saber, conforme a los principios o actitudes del perfil y de la 
Revista de la Escuela de Ciencias de la Educación, año 11, número 10, enero a diciembre de 2015. Páginas 111-130. ISSN 1851-6297. ISSN EN LINEAA 2362-3349. LOS SABERES DOCENTES EN LA MIRA: UNA APROXIMACIÓN POLIFÓNICA. ANDREA Alliaud - Lea Vezub

identidad profesional que están formando: flexibilidad, complejidad, apertura, carácter integral, dinamismo, provisionalidad del saber.

"Un saber didáctico que muchas veces es complejo, que tiene que poder ser flexible para poder adaptarse a los nuevos desarrollos teóricos, estar abierto a que esos cambios los puedan ir incorporando" (F5).

Entre estas características una mención especial ocupa desde la perspectiva de los formadores, la capacidad de relacionar la teoría con la práctica. Según los entrevistados, la formación se orienta a producir esa intersección, interjuego o entramado. Lo que se estudia en la formación se tiene que actuar. Una profesora plantea que si se trabaja a nivel teórico la escuela inclusiva y que todos los alumnos son sujetos de derecho, el problema es luego ver cómo se traducen esas ideas en determinadas prácticas, estrategias, formas de enseñar y de vincularse con los estudiantes: "el tema es cómo actúan". Es justamente en el terreno, en la acción, donde se identifican las dificultades.

"No sólo es importante la disciplina, sino el enfoque didáctico, para mí son los puntos básicos. El saber didáctico particular de cómo leer la clase, a ver los pibes, cómo circula el conocimiento en la clase, ese saber es muy importante. (...) En los talleres de práctica hay que analizar cómo viste que el grupo aprendió, cuántos no, y cómo se lee esa información para ver qué se cambia, cómo ves la evaluación, qué se puede modificar (...) hay otros espacios de la formación que tienen que ver con la formación general como Pedagogía, Historia de la Educación, Didáctica... hay que ver en qué momento el alumno cruza esto con el campo de la práctica que tiene que ser articuladora" (F13).

Una serie de saberes vinculados con las habilidades, estrategias propias de la transmisión y gestión de los grupos de estudiantes son ubicados, por los formadores consultados, dentro de un grupo específico que comienza a denominarse y asumir su propia identidad para algunos formadores. Se trata de los "conocimientos de la práctica profesional", de un estar ahí, en situación:

"Salir de la lógica de que tener una secuencia de actividades es poder enseñar. Este, poder tomar conciencia ahí y en situación de tener que dar clase también, de poder pensar en todas las decisiones que están en torno al momento de empezar a trabajar sobre un tema con los alumnos..." (F11).

"Conocimientos que tienen que ver con todas las cuestiones pedagógicas, didácticas por decirlo de otra manera, y conocimientos de la práctica profesional. $Y$ en esto me parece que cada vez los campos son más amplios" (F3). 
Revista de la Escuela de Ciencias de la Educación, año 11, número 10, enero a diciembre de 2015. PÁginas 111-130. ISSN 1851-6297. ISSN EN LINEA 2362-3349. LOS SABERES DOCENTES EN LA MIRA: UNA APROXIMACIÓN POLIFÓNICA. ANDREA AlLiaud - Lea VezuB

En este sentido estos formadores consideran importante recuperar los saberes de la propia experiencia profesional docente para poder sistematizarlos y compartirlos, aunque se trata de conocimientos generalmente no reconocidos o valorados:

"Saberes que tienen que ver por ahí no tanto con la formación académica... estos saberes que nosotros los ponemos en un escalón más bajo, que no son saberes teóricos ni didácticos, que tienen que ver con capitalizar la experiencia, con saberes propios que va generando el mismo maestro en su ejercicio docente, que tienen que ver con esto del oficio. Yo les digo mucho a mis alumnos "escriban, escriban sus prácticas", escriban porque es un saber que lo están construyendo día a día y esto el día de mañana va a ser parte de su acerbo más allá de los libros, más allá de lo que puedas ir encontrando de recetas que te van pasando, lo que vos vas construyendo en tu experiencia es invaluable" (F5).

El saber relacionado con el contexto y la posibilidad de constituir aulas democráticas e inclusivas, significa volver la mirada sobre las características de los sujetos de la educación, analizar los rasgos de las infancias y adolescencias actuales, con la idea de que los futuros docentes puedan: "mirar a los chicos o adolescentes con una cabeza, con ciertas herramientas teóricas que permitan ayudar a comprender y acercarse a esa realidad" (F11). Pero también supone un docente informado, "sensible" al contexto, conectado con la sociedad y el mundo que habita.

"Las situaciones de enseñanza son situadas y contextualizadas, por lo tanto el contexto se debe conocer. Tendría que haber algún tipo de saberes relacionados con lo que se denomina "insumos culturales", un docente tendría que poder tener acceso o tener algún espacio de formación donde saber que tiene que estar informado, en distintas cosas, lo que pasa en el día, hoy, en el mundo, no sé... hoy se entregó el Premio Nobel de Química, un profesor de Química no puede mínimamente no saber que hoy se entregó el Premio Nobel de Química y cuál es el tema por el cual se entregó. Digamos, como información, más allá de otros insumos culturales que también debe haber. La problemática del conocimiento del sujeto con el que uno está trabajando... La reconfiguración de los campos familiares hoy por hoy es terrible, y creo que un docente no está preparado..." (F3).

Por último, los formadores les dan gran importancia a los saberes que de manera clásica e históricamente estuvieron asociados al ejercicio de la docencia: el saber disciplinar, establecer vínculos, comunicarse, las 
Revista de la Escuela de Ciencias de la Educación, año 11, número 10, enero a diciembre de 2015. Páginas 111-130. ISSN 1851-6297. ISSN EN LINEAA 2362-3349. LOS SABERES DOCENTES EN LA MIRA: UNA APROXIMACIÓN POLIFÓNICA. ANDREA AlLiaud - Lea Vezub

herramientas didácticas y pedagógicas (planificación, manejo de recursos, estrategias de enseñanza).

"Aunque suene redundante saber disciplinar, hay muchos docentes y muchos lugares de formación donde no está lo disciplinar (...) Eso por una parte como el conocimiento básico" (F3).

"Los rudimentos de lo que es el saber didáctico, pero básicamente, la enseñanza de cada disciplina (...) el saber didáctico que acompaña cómo se enseña cada asignatura" (F5).

"Saberes que son específicos de las didácticas y que son necesarios para poder acompañar los procesos de enseñanza... Y otras cuestiones que hacen al rol docente y a la tarea pedagógica. El posicionamiento del adulto, la construcción de legalidades en la escuela, lo normativo, lo ético... el rol docente como autoridad, como posibilidad de acercar a los chicos a la cultura (...) el armado y la planificación de secuencias didácticas y luego, su implementación" (F11).

"Más intensificación en las áreas de enseñanza, me refiero a las áreas curriculares, Matemática, Ciencias naturales, Sociales, Prácticas del Lenguaje, etc." (F1).

\section{Conclusiones}

Inmersos en la práctica de enseñar indagamos, desde allí, los saberes puestos en juego por los docentes. Incorporamos las voces de los especialistas y de los formadores, a fin de avanzar en propuestas formativas que las contemplen con la finalidad de preparar docentes que puedan y sepan enseñar en el presente. Al contrastar las voces de los distintos actores relevadas en el estudio, se manifiestan similitudes y diferencias. En los tres casos se valoriza la dimensión "clásica" del oficio del enseñar y los saberes específicos (disciplinares, didácticos y pedagógicos) implicados. Los conocimientos acerca del contexto, de las nuevas tecnologías y fundamentalmente de los alumnos en su faceta, no sólo cognitiva, sino también social y cultural, así como la atención a la diversidad y a la diferencia, parecen ser necesarios para responder a los nuevos desafíos que atraviesa la docencia. Al igual que lo establecido por los expertos, las respuestas brindadas por los docentes y formadores consultados dan cuenta de que el conocimiento del contexto y de los alumnos es central para afrontar el trabajo de enseñar en la actualidad.

Si bien se detectaron estas coincidencias, también se observan diferencias en las prioridades que cada uno le atribuyen a la dimensión "clásica" del oficio. Mientras los formadores le otorgan una importancia "crucial", no parece tener el mismo peso o ser un saber que "urge", para los docentes. Es interesante notar aquí que la fuente de validez acerca de los saberes que los docentes conside- 
Revista de la Escuela de Ciencias de la Educación, año 11, número 10, enero a diciembre de 2015. PÁginas 111-130. ISSN 1851-6297. ISSN EN LINEA 2362-3349. LOS SABERES DOCENTES EN LA MIRA: UNA APROXIMACIÓN POLIFÓNICA. ANDREA AlLiaud - Lea VezuB

ran útiles o valiosos, descansa en el puesto de trabajo, en la experiencia y el trato con los alumnos. Los docentes hablan desde "su" experiencia y desde allí cobra protagonismo otro tipo de saberes que ni los formadores, ni los especialistas, mencionan $\mathrm{o}$, si lo hacen, no les otorgan la importancia que tiene para sus protagonistas. Maestros y profesores valoran fundamentalmente los saberes que les permiten "poder hacerlo", estos son, por ejemplo: despertar la motivación y el compromiso de los chicos, poder acompañarlos y adaptar las herramientas pedagógicas a sus características, tener en cuenta sus saberes previos, saber escucharlos, ponerse en su lugar y crear un buen vínculo, lograr una buena dinámica en la clase, guiarlos, darles confianza, enseñarles a ser persistentes, estimular su participación, etc.

Los docentes consultados ponen la atención en el alumno, pero cuando lo hacen se refieren más que al logro de su aprendizaje, a la posibilidad de motivarlo, entusiasmarlo, incentivarlo; suponiendo que desde allí luego, es posible enseñar y aprender. Es frecuente la referencia a la motivación como condición primera, anterior, que precede a la enseñanza. Quizás el problema lo constituya donde se centra el foco: si estamos enfocados en la enseñanza (centro nodal de nuestro oficio, que le da vida y sentido) es probable que los alumnos "se enganchen", se motiven y entusiasmen, aún sin quererlo o, mejor, queriéndolo, aprendan. No es por pasos, ni de manera separada o disociada que es posible lograrlo; al enseñar se puede motivar, entusiasmar y también aprender. Aunque no hay que olvidar que el que manda en educación es siempre el otro. En este sentido, el aprendizaje es una decisión y que así sea es señal de que estamos vivos y no un obstáculo que debe ser despejado (Cf. Alliaud y Antelo, 2008).

El saber sobre el contexto, también aparece recurrentemente en las distintas voces relevadas. En principio parecería que se trata, no de un saber clásico del oficio, sino de un saber ligado a las nuevas demandas profesionales, a la necesidad de adaptar la enseñanza al otro, de recibir a nuevos sujetos, de incluir a todos, de atender la diversidad cultural del aula. Por lo tanto, un saber vinculado con la expansión de los sistemas educativos. No obstante cabría una reflexión similar a la anterior en cuanto a cuál debe ser el foco. Si el contexto es tan importante y decisivo podríamos pensar que una solución sería formar docentes con distintos perfiles, saberes y habilidades profesionales, de acuerdo al contexto en que vayan a desempeñarse. ¿Maestros para escuelas difíciles, más o menos fáciles, urbanas, marginales? ¿Se requieren distintos saberes para cada contexto, según su grado de dificultad, complejidad, características o tipo de poblaciones escolares? No creemos que esa sea la alternativa. ¿O será que los saberes son los mismos pero su grado de dominio y expertez debe variar según los contextos? Contrariamente a lo que suele ocurrir en la dinámica del puesto de trabajo, apostamos a que docentes más experimentados y más sabios, con mayor pericia y oficio se desempeñen en los contextos más 
Revista de la Escuela de Ciencias de la Educación, año 11, número 10, enero a diciembre de 2015. Páginas 111-130. ISSN 1851-6297. ISSN EN LINEAA 2362-3349. LOS SABERES DOCENTES EN LA MIRA: UNA APROXIMACIÓN POLIFÓNICA. ANDREA Alliaud - Lea Vezub

difíciles y complejos, donde serán capaces de generar mejores condiciones para el aprendizaje.

Por último, a partir de los resultados obtenidos podemos esbozar algunos criterios para repensar las propuestas en los ámbitos formativos. Si bien es necesario sumar nuevos conocimientos formales, dotarlos de mayor complejidad y profundidad durante la formación, también se requiere que esos saberes sean puestos al servicio de situaciones de enseñanza, de producciones, de creaciones docentes. Es decir, se precisa construir el oficio de enseñar a lo largo de todo el proceso formativo, mediante formas de formar que no disocien el saber del hacer, la teoría de la práctica, el pensamiento de la acción. $Y$ para eso tenemos que apelar y convocar muchas y variadas experiencias de enseñanza que nos impliquen, que den lugar a un nuevo saber, que nos convoquen a abrirnos a lo que sucede, a pensar, a inventar (Alliaud, 2013). Las narraciones pedagógicas, las "obras de enseñanza”, las conversaciones con docentes experimentados, las historias de clases bien y mal avenidas, junto con otras formas más tradicionales, pero no aún del todo usuales en la formación como los ateneos, talleres, estudios de casos o el acompañamiento, resultan propicias para explorar esos abismos que se generan entre la teoría y la práctica, para habilitar nuevas maneras de pensamiento y acción, para interpelar desde el hacer, al saber formalizado.

\section{Notas Bibliográficas}

(1) Si bien mucho se ha debatido en torno al término "transmisión", coincidimos con las visiones actuales, como las expresadas por Meirieu, entre otros, para quienes la transmisión no se asocia a la enseñanza tradicional, ni a una concepción pasiva del sujeto de conocimiento. Por el contrario, todo proceso de enseñanza y formación requiere de actividades de transmisión junto con otras de apropiación, significación, elaboración personal e idiosincrásica que despliega la actividad cognitiva y experiencial de cada individuo.

(2) Una excepción a esta forma de elaborar una propuesta de formación docente, se encuentra en la obra de Perrenoud (2007), quien basa su planteo de nuevas competencias para enseñar en el estudio de las prácticas docentes y en el referencial adoptado en Ginebra (1996) para la formación continua, con la intención de que sea un instrumento para pensar las prácticas y debatir sobre la profesión. Sin entrar en la discusión acerca de la pertinencia de este tipo de formulaciones, se destaca la preocupación del autor por considerar las características del desempeño docente (los problemas y las tareas) para pensar la formación.

(3) "El saber de la experiencia. Experiencias pedagógicas, narración y subjetividad en la trayectoria profesional de los docentes", Subsidio UBACYT 2004 - 2007. "Pedagogos, docentes y relatos de experiencia. Saberes pedagógicos y experiencias formativas producidas en la trayectoria profesional. Subsidio UBACYT 2008-2010. "Dimensiones del saber profesional docente. Su estudio y aportes a la formación". Subsidio UBACYT 2011 - 2014. IICE, Facultad de Filosofía y Letras, UBA. Directora: Dra. Andrea Alliaud. 
Revista de la Escuela de Ciencias de la Educación, año 11, número 10, enero a diciembre de 2015. PÁginas 111-130. ISSN 1851-6297. ISSN EN LINEA 2362-3349. LOS SABERES DOCENTES EN LA MIRA: UNA APROXIMACIÓN POLIFÓNICA. ANDREA AlLIAUd - LEA VEZUB

(4) Se administró un cuestionario estructurado a una muestra intencional de 263 docentes de nivel primario y secundario en la Ciudad de Buenos Aires y tres distritos del Conurbano Bonaerense entre septiembre y noviembre de 2011.

(5) Se seleccionaron de la muestra anterior 17 maestros y profesores a los que se les realizó una entrevista en profundidad en marzo de 2013. Posteriormente se completó el trabajo de campo con 8 entrevistas a formadores de Institutos de CABA entre agosto y septiembre de 2013.

\section{Referencias Bibliográficas}

- $\quad$ Alliaud, A. y Antelo, E. (2008). "El fracaso de enseñar. Ideas para pensar la enseñanza y la formación en los futuros docentes". En: Braislovsky, D. Coord. Sentidos perdidos de la experiencia escolar. Buenos Aires: Noveduc.

- $\quad$ Alliaud, A. (2013). "Formar buenos en la artesanía de enseñar". En: VIII Foro Latinoamericano de Educación. Saberes Docentes: qué debe saber un docente y por qué. Buenos Aires: Fundación Santillana. http://www.fundacionsantillana.com/upload/ficheros/ noticias/201304/8vo_foro_baja.pdf

- $\quad$ Alliaud, A. (2013). "El saber disponible parece no ser suficiente: acerca de las formas de producción y circulación del saber pedagógico". Primer Congreso Provincial de Formación Docente Continua de la Provincia de Buenos Aires. Revista Anales de Educación, La Plata: DGCyE de la Provincia de Buenos Aires, en prensa.

- $\quad$ Alliaud, A. y Vezub, L. (2012). "El oficio de enseñar: sobre el quehacer, el saber y el sentir de los docentes argentinos". Revista Diálogo Educacional, Vol. 12, Núm. 37, 927-952. Pontifícia Universidade Católica do Paraná, Curitiba, Brasil. Disponible en: http://www2. pucpr.br/reol/index.php/dialogo?dd1=7211\&dd99=view

- $\quad$ Antelo, E. Coord. (2009). ¿Qué sabe el que sabe enseñar? Un estudio exploratorio acerca del saber de los profesores en la escuela secundaria. Dirección de Investigación y estadística, Ministerio de Educación, Gobierno de la Ciudad Autónoma de la Ciudad de Buenos Aires.

- Ávalos, B. (2009). "Los conocimientos y las competencias que subyacen a la tarea docente". En: Vélaz de Medrano, C. y Vaillant, D. Aprendizaje y Desarrollo Profesional. Metas Educativas 2021. Madrid: Fundación Santillana - OEl.

- Braslavsky, C. (1999). Re-haciendo escuelas. Hacia un nuevo paradigma en la educación latinoamericana. Buenos Aires: Santillana - Andrés Bello.

- Bromme, R. (1988). "Conocimientos profesionales de los profesores". Enseñanza de las ciencias, 6 (1), 19-29.

- $\quad$ Calderhead, J. (1988). "Conceptualización e investigación del conocimiento profesional de los profesores”. En: Villar Angulo M. (Dir.). Conocimiento, creencias y teorías de los profesores. Alcoy: Editorial Marfil.

- Dubet, F. (2006). El declive de la institución. Profesiones, sujetos e individuos en la modernidad. Barcelona: Gedisa.

- $\quad$ Elbaz, F. (1983). Teacher Thinking: A study of practical knowledge. New York: Croom Helm - Nichols Publishing Company.

- Gimeno Sacristán, J. (1997). Docencia y Cultura escolar. Buenos Aires: IDEAS.

- Hassoun, J. (1996). Los contrabandistas de la memoria. Buenos Aires: Ediciones de la Flor.

- $\quad$ Pendelbury, S. (1998). "Razón y relato en la buena práctica”. En: McEwan, H. y Egan, K. La narrativa en la enseñanza, el aprendizaje y la investigación. Buenos Aires: Amorrortu. 
Revista de la Escuela de Ciencias de la Educación, año 11, número 10, enero a diciembre de 2015. Páginas 111-130. ISSN 1851-6297. ISSN EN LINEA 2362-3349. LOS SABERES DOCENTES EN LA MIRA: UNA APROXIMACIÓN POLIFÓNICA. ANDREA AlLIAUd - Lea VeZub

- $\quad$ Perrenoud, Ph. (2007). Diez nuevas competencias para enseñar. Barcelona: Graó.

- $\quad$ Sennett, R. (2009). El artesano. Barcelona: Anagrama.

- Schön, D. (1992). La formación de profesionales reflexivos. Hacia un nuevo diseño de la enseñanza y el aprendizaje en las profesiones. Barcelona: MEC - Paidós.

- $\quad$ Schulman, L. (1987). Knowledge and teaching: Foundations of de New Reform. Harvard Educational Review, Vol. 57, Núm.1, 1-22.

- Tardif, M. (1991). Les enseignants des ordres d'enseignement primaire et secondaire face aux savoirs. Esquisse d'une problématique du savoir enseignant. Sociologie et societés. Vol. XXIII, N¹, 55-69.

- Tardif, M. (2000). Saberes profissionais dos profesores e conhecimientos universitarios. Revista Brasilera de Educación N¹3, 5-24.

- Terigi, F. (2009). "La formación inicial de profesores de Educación Secundaria: necesidades de mejora, reconocimiento de sus límites". Revista de Educación, №350, 123-144. Madrid: MEC.

- Terigi, F. (2012). Los saberes docentes. Formación, elaboración en la experiencia e investigación. Documento Básico VIII Foro Latinoamericano de Educación, Buenos Aires: Fundación Santillana.

- Vezub, L. (2011). "¿Qué cuentan las trayectorias de desarrollo profesional de los docentes sobre su oficio?” En: Alliaud, A. y Suárez, D. (comps.) El saber de la experiencia. Narrativa, investigación y formación docente. Buenos Aires: Facultad de Filosofía y Letras, UBA - CLACSO. 